\title{
Avaliação do perfil clínico e prática de atividades físicas em idosos diabéticos, cadastrados na Estratégia Saúde da Família
}

\author{
Evaluation of clinical profile and physical activity habits of diabetic elderly, under Family \\ Health Strategy users
}

\section{Evaluación del perfil clinico y la práctica de las actividades físicas en los ancianos con diabetes registrados en la Estrategia de Salud Familiar}

Rodrigo Mendes de Freitas ${ }^{1 *}$, Virna Borém Valle Pereira ${ }^{1}$, Matheus Mendes Cotrim ${ }^{1}$, Eduardo Gonçalves ${ }^{1}$, Andréa Maria Eleutério de Barros Lima Martins ${ }^{2}$.

\section{RESUMO}

Objetivo: Analisar a percepção da importância e da efetiva prática de atividades físicas entre idosos com diabetes mellitus, cadastrados na Estratégia Saúde da Família. Métodos: Trata-se de um estudo descritivo, transversal e de abordagem quantitativa. Utilizou-se o International Physical Activity Questionnaire (IPAQ) e autorrelatos para a coleta dos dados. Estes foram separados em categorias: sedentário, insuficientemente ativo, ativo e muito ativo. Resultados: Foram envolvidos 198 idosos cadastrados em 6 Estratégias Saúde da Família, de ambos os sexos, com idades entre 60 e 92 anos. Constatou-se que, dos 198 idosos entrevistados, há uma discrepância entre o número total de pessoas ativas de forma insatisfatória (Sedentários e Insuficientemente ativos A e B), correspondendo a 85,05\% (169), e o número de pessoas com desempenho satisfatório (Ativo e Muito Ativo), com 14,95\% (29), mesmo que 76,3\% (151) desses tenham sido atualizados, nos últimos 06 meses, sobre os benefícios das atividades físicas. Conclusão: A maioria dos idosos foi categorizado como insuficientemente ativo. Logo, praticam atividades físicas durante a semana regularmente, mas insuficientemente para serem ativos. Portanto, torna-se necessário aprimorar a cobertura da atenção primária a respeito da prática de exercícios físicos, visando encorajá-los a exercitar-se, de forma coerente com a capacidade individual e com o seu estado de saúde.

Palavras-chave: Atividade física, Diabetes mellitus, Idosos, Perfil.

\section{ABSTRACT}

Objective: Analyze the perception and the importance of the effective practice of physical activities by seniors with diabetes mellitus under Family Health Strategy. Methods: It's a descriptive, transverse and quantitative study. It was used the International Physical Activity Questionnaire (IPAQ) and self-reports for the data collect. It were splitted in categories: sedentary, insufficient active, active and very active. Results: Were envolved 198 seniors registered in 6 Family Strategy od Health, both genders, ages between 60 and 92 years. It was founded that, from the 198 interviewed seniors, there was a discrepancy among the total number of active people with a unsatisfactory manner (sedentaries and unsatisfactory actives $A$ and $B$ ), corresponding a $85,05 \%$ (169), and the number of people with satisfactory performance (active and very active), in 14,95\% (29), even that $76,3 \%$ (151) from these has been updated, in the last 06 months, about the benefits of the physical activites. Conclusion: Most of the analyzed seniors is insufficient active. Therefore, it becomes necessary to improve the the covering of the primary attention about the practice of physical activities.

Keywords: Physical activity, Diabetes Mellitus, Seniors, Profile.

\section{RESUMEN}

Objetivo: Analizar la percepción de la importancia y la práctica efectiva de las actividades físicas en ancianos con diabetes mellitus registrados em la Estrategia de Salud Familiar. Métodos: Es un estudio descriptivo, transversal con un enfoque cuantitativo. El International Physical Activity Questionnaire (IPAQ) y los autoinformes se utilizaron para la recopilación de datos. Estos se dividieron en categorías: sedentarios, insuficientemente activos, activos y muy activos. Resultados: Participaron un total de 198 ancianos diabéticos

\footnotetext{
${ }^{1}$ Centro Universitário FIPMoc (UNIFIPMOC), Montes Claros - MG. *E-mail: rodrigomendes2009@yahoo.com.br

${ }^{2}$ Pós-Graduação em Ciências da Saúde da Universidade de Montes Claros (Unimontes), Montes Claros - MG.
} 
inscritos en 6 Estrategia de Salud Familiar, de ambos sexos, de 60 a 92 años. De los 198 ancianos, existe una discrepancia entre el número total de activos insactisfactorias (sedentarias e insuficientemente activas $A$ y B), que corresponde al 85,05\% (169), y el número de personas con rendimento satisfactorio (activo e muy activo), en el 14,95\% (29), aunque, el 76,3\% (151) de estos se ha actualizado en menos de 6 meses sobre los beneficios de la actividad física. Conclusión: La mayoría de los ancianos analizados son insuficientemente activos. Por lo tanto, hacen ejercicio durante la semana regularmente, pero insuficientemente para estar activos. Por lo tanto, es necesario mejorar la cobertura de la atención primaria con respecto a la práctica de ejercicios físicos.

Palabras clave: Actividad física, Diabetes Mellitus, Ancianos, Perfil.

\section{INTRODUÇÃO}

O diabetes mellitus (DM) é um distúrbio metabólico que atinge crianças, jovens, adultos e idosos (MALTA DC, et al., 2017). O Brasil é o quarto país com maior prevalência da patologia cerca de 14,3 milhões de pessoas foram acometidas pelo DM. A estimativa é que em 2040 o Brasil possua 23,3 milhões de pessoas portadoras da doença. Tal ascensão, pode ser explicada pelo aumento da exposição das pessoas a fatores de risco, tais como idade maior que 50 anos, obesidade, hipertensão arterial sistêmica e aumento dos níveis séricos de colesterol (SANTOS LM, 2017). Ele pode ser classificado como tipo 1 (DM1), no qual as manifestações ocorrem de forma precoce, geralmente ainda na primeira década de vida, com a deficiência completa da produção de insulina endógena. Há também o tipo 2 (DM2), que representa cerca de $90 \%$ do total de DM, característico da meia-idade e pode ser inicialmente tratado com medicações de sensibilização e incremento da produção da insulina (FILHO CAAD, et al., 2016). Nos idosos, comumente expostos por maior tempo ao DM, observa-se a perda funcional das células $\beta$ associada ao aumento da adiposidade central, diminuição da massa magra, aumento da resistência à insulina (que por si resulta em redução do tecido muscular) e predisposição a morbidades, que elevam o risco de quedas e fraturas, eventos cardiovasculares e perda da autossuficiência (RAMOS RSPS, et al., 2017).

Trata-se, portanto, de uma síndrome metabólica de caráter multifatorial, que apresenta complicações agudas como hipoglicemia; cetoacidose diabética - condição mais prevalente no DM1-; estado hiperosmolar hiperglicêmico não cetótico - condição mais prevalente no DM2-; bem como complicações crônicas, que podem ser macrovasculares (doença arterial coronariana e cerebrovascular) ou microvasculares (neuropatia, nefropatia e retinopatia) (SANTOS LM, et al., 2017). Nos idosos, o DM associa-se a um risco maior de morte prematura e outras morbidades, principalmente, as grandes síndromes geriátricas, impactando diretamente na qualidade de vida das pessoas com DM, que terão prejuízo em suas capacidades funcionais, independência e autonomia, repercutindo sobre o sistema de saúde, e sobre a família das pessoas com DM (VITOI NC, et al., 2015). É uma doença que causa manifestações complexas e heterogêneas; sua consequência é expressar-se com elevada morbimortalidade (ISER BPM, et al., 2015). Foi estimado que 5,3\% dos óbitos ocorridos em 2011 no Brasil foram devido à DM, com taxa de mortalidade por volta de 33,7 a cada 100.000 habitantes. Atualmente é a nona causa de morte no mundo, entretanto, até 2030 , o DM pode ir para a sétima posição (FLOR LS e CAMPOS MR, 2017).

O sedentarismo aumenta o risco na prevalência do DM tipo 2, por isso as populações de risco e as pessoas já diagnosticados devem implantar a prática de atividade física em seu plano terapêutico como medida de controle glicêmico/diminuição da resistência insulínica, evitando deste modo complicações do DM e melhorando a qualidade de vida das pessoas (DE LADE CG, et al., 2016). Entre idosos, além dos benefícios já citados, a atividade física é importante para a manutenção da massa magra, ajudando também na prevenção e no tratamento da sarcopenia, redução do risco de mortes prematuras, de doenças cardiovasculares, do ganho de peso ponderal (diminuindo o risco de obesidade), bem como auxiliando na prevenção/redução da osteoporose e promovendo bem-estar (MAZO GZ, 2018). Os exercícios aeróbicos e de resistência, se praticados de forma contínua durante pelo menos 20 semanas, auxiliam no controle glicêmico nas pessoas portadoras de DM, período suficiente para alterar a glicemia média estimada e a hemoglobina glicada (COLBERG SR, et al., 2016). Portanto, a atividade física é um pilar importante para o tratamento não farmacológico do DM, de forma independente da idade, com impacto no controle glicêmico e no controle das morbidades frequentemente associadas ao DM, como a hipertensão arterial sistêmica, a dislipidemia e a síndrome metabólica (KER RG, 2018). 
Ao observar a relevância das medidas de estilo de vida, a adoção da prática dos exercícios físicos nas pessoas com DM, principalmente nos idosos, nota-se efetivo controle glicêmico e controle de morbidades. A abordagem do tema sobre as atividades físicas foi baseada em sua eficácia e indicação como tratamento coadjuvante entre pessoas com DM, que devem ser estimuladas a permanecer tão ativas quanto a saúde permitir. Objetivou-se apresentar descritivamente o conhecimento e a efetiva prática de atividades físicas entre idosos com DM, bem como o perfil clínico-epidemiológico e sócio demográfico desses idosos, viabilizando assim o estabelecimento de estratégias de controle e intervenção efetiva na vida das pessoas com DM, à medida que seja estimulada a disseminação da informação e a prática de atividades físicas de forma adequada.

\section{MÉTODOS}

Estudo descritivo com delineamento transversal, de abordagem quantitativa realizado no período de janeiro de 2017 a dezembro de 2017, em seis unidade de saúde da Estratégia de Saúde da Família (ESF) em uma cidade do interior de Minas Gerais, após seleção por método de amostragem aleatória simples das seis unidades dentre as 137 unidades de saúde do município. Foram avaliados198 idosos portadores de DM. O estudo foi submetido e aprovado pelo Conselho de Ética da Universidade Estadual de Montes Claros Unimontes, sob o parecer n: 764.743 , seguindo os princípios éticos definidos pela Resolução 466/2012, do Conselho Nacional de Saúde, para realização de pesquisas que envolvem seres humanos.

Os dados foram coletados com por meio de entrevistas e utilização de um questionário baseado no relato dos idosos com DM, contendo variáveis sociodemográficas, econômicas e indicadores do nível de conhecimento e da efetiva prática de atividades físicas. A avaliação da prática de atividade física considerou o International Physical Activity Questionnaire (IPAQ), versão longa, com validação em nível nacional e internacional (MATSUDO S, et al., 2001). As outras variáveis avaliadas foram: sexo, idade, tipo do diabetes, raça e renda familiar. Avaliou-se ainda o conhecimento dos idosos frente à importância da prática de atividades físicas, os meios pelos quais adquiriram as informações referentes à importância da prática de atividade física e há quanto tempo se atualizaram acerca deste tema.

O IPAQ estabelece o perfil e quantifica, de maneira eficiente e econômica, o nível de atividade física da população em estudo. Permite estimar o tempo semanal, variando desde intensidade leve à vigorosa, divididas em 04 sessões que englobam a rotina diária: trabalho; transporte; atividades domésticas; e atividades de recreação e lazer. Há ainda uma sessão específica para estimar o tempo despendido em atividades passivas realizadas na posição sentada. $O$ instrumento é aceito para estudos populacionais, delimitando as atividades menos e mais ativas (MATSUDO S, et al., 2001). Ao fim da coleta, os dados informados foram avaliados e dispostos conforme a adaptação brasileira para o IPAQ, proposta pelo CELAFISCS (Centro de Estudos do Laboratório de Aptidão Física de São Caetano do Sul), que classifica os indivíduos em 5 categorias (Quadro 1).

Quadro 1 - Classificação do nível de atividade física pelo IPAQ.

\begin{tabular}{|c|c|}
\hline$\underline{\text { Sedentário }}$ & $\begin{array}{l}\text { Não realiza nenhuma atividade física em tempo mínimo de } 10 \text { minutos } \\
\text { contínuos durante a semana. }\end{array}$ \\
\hline \multirow[t]{2}{*}{$\frac{\text { Insuficientemente ativo }}{\text { (divido em } 2 \text { subcategorias) }}$} & $\begin{array}{l}\text { Tipo }(\mathbf{A}) \text { : Realiza pelo menos } 10 \text { minutos contínuos de atividade física, } \\
\text { seguindo ao menos um dos critérios citados: } \\
\text { Frequência }-5 \text { dias /semana } \\
\text { ou } \\
\text { Duração }-150 \text { minutos /semana; }\end{array}$ \\
\hline & $\begin{array}{l}\text { Tipo (B): Pratica pelo menos } 10 \text { minutos, mas não preenche nenhum } \\
\text { critério supracitado. }\end{array}$ \\
\hline \multirow{3}{*}{$\begin{array}{c}\text { (pessoa que preenche } 1 \text { dos } 3 \\
\text { critérios) }\end{array}$} & \\
\hline & MODERADA ou \\
\hline & Qualc \\
\hline \multirow{2}{*}{$\begin{array}{c}\text { Muito ativo } \\
\begin{array}{c}\text { (pessoa que preenche } 1 \text { dos } 2 \\
\text { critérios) }\end{array}\end{array}$} & Atividade VIGOROSA: $\geq 5$ dias /semana e $\geq 30$ min / sessão; \\
\hline & $\begin{array}{l}\text { Atividade VIGOROSA: } \geq 3 \text { dias } / \text { semana e } \geq 20 \mathrm{~min} / \mathrm{sessão} \mathrm{E} \text { mod } \\
\text { e/ou caminhada } \geq 5 \text { dias } / \text { semana e } \geq 30 \mathrm{~min} / \mathrm{sessão}\end{array}$ \\
\hline
\end{tabular}

Fonte: Freitas RM, et al., 2019. Adaptado de Silva GSF, et al. 2007. 
Para a análise final, fez-se uso da estatística descritiva para discorrer acerca do perfil sociodemográfico e do nível de conhecimento e da efetiva prática de atividades físicas, relatados pelos idosos. Foi estimada a distribuição percentual da amostra em relação às categorias propostas pelo instrumento. Variáveis quantitativas foram apresentadas por meio das estimativas da média aritmética. Utilizou-se o programa estatístico Statistical Package for Social Sciences ${ }^{\mathrm{TM}}$ (SPSS), versão 21.

\section{RESULTADOS}

Para melhor análise dos perfis dos pacientes diabéticos, frente à diversidade encontrada entre os 341 pacientes envolvidos, a tabela abaixo foi construída com base em variáveis para demonstrar as características dos pacientes. Dessa forma, foi selecionado as variáveis sexo, idade, tipo do diabetes, cor/raça e renda mensal (Tabela 1).

Tabela 1 - Perfil dos idosos com Diabetes Mellitus assistidos pela Estratégia de Saúde da Família, n=198. Montes Claros - MG, 2017.

\begin{tabular}{|c|c|c|}
\hline Variável & $\mathbf{n}$ & $\%$ \\
\hline \multicolumn{3}{|l|}{ Sexo } \\
\hline Masculino & 62 & 31,417 \\
\hline Feminino & 136 & 68,582 \\
\hline \multicolumn{3}{|l|}{ Idade } \\
\hline $60-65$ & 100 & 50,50 \\
\hline $65-69$ & 39 & 19,54 \\
\hline $70-74$ & 28 & 14,17 \\
\hline $75-79$ & 15 & 7,27 \\
\hline $80-84$ & 12 & 6,13 \\
\hline $85-90$ & 3 & 1,53 \\
\hline $91-95$ & 1 & 0,51 \\
\hline \multicolumn{3}{|l|}{ Cor/Raça } \\
\hline Amarela & 2 & 1,140 \\
\hline Branca & 51 & 26,05 \\
\hline Indígena & 1 & 0,510 \\
\hline Parda & 109 & 55,255 \\
\hline Preta & 27 & 14,171 \\
\hline Sem declaração & 8 & 4,19 \\
\hline \multicolumn{3}{|l|}{ Renda em reais } \\
\hline $0-1000$ & 90 & 45,59 \\
\hline $1001-2000$ & 65 & 32,56 \\
\hline $2001-3000$ & 25 & 12,64 \\
\hline $3001-4000$ & 10 & 5,36 \\
\hline $4001-5000$ & 7 & 3,44 \\
\hline $5001-10000$ & 1 & 0,51 \\
\hline \multicolumn{3}{|l|}{ Diabetes mellitus * } \\
\hline Tipo 1 & 9 & 4,4 \\
\hline Tipo 2 & 189 & 95,6 \\
\hline Total & 198 & 100 \\
\hline
\end{tabular}

Legenda: * Dados correspondem às estimativas das Diretrizes da Sociedade Brasileira de Diabetes (DSBD) 2017-2018. Fonte: Freitas RM, et al., 2019.

Foram analisados ainda os relatos do nível de conhecimento e da efetiva prática de atividades físicas entre os participantes (Gráfico 1). 
Gráfico 1 - Avaliação do nível de conhecimento e da efetiva prática de atividades físicas entre idosos assistidos pela Estratégia de Saúde da Família, n=198. Montes Claros - MG, 2017.

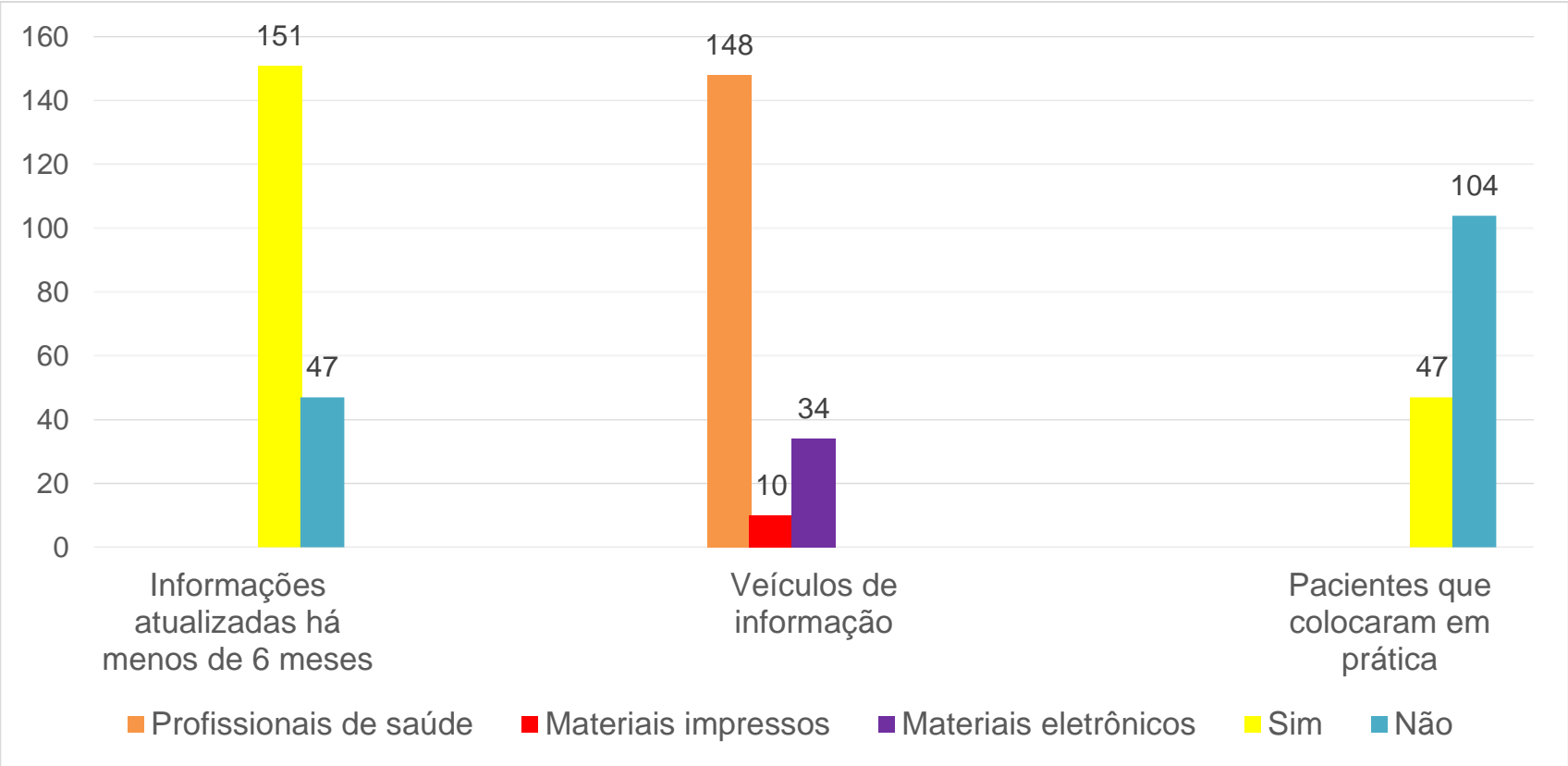

Fonte: Freitas RM, et al., 2019.

Dos 198 participantes, apenas 49 possuem trabalho ativo. Dentre os trabalhadores, a maioria realiza atividades moderadas por pelo menos 10 minutos, de modo que gastam uma média de 80 minutos ao dia, durante 4,3 dias na semana (Tabela 2).

Tabela 2 - Caracterização da atividade física não recreativa, $n=198$ entre idosos assistidos pela Estratégia de Saúde da Família, Montes Claros - MG, 2017.

\begin{tabular}{lrrr}
\hline Variável & N & \multicolumn{1}{l}{$\%$} & Tempo médio gasto/dia \\
\hline (01) Pessoas que trabalham & & & - \\
\hline Sim* & 49 & 24,9 & -- \\
Não & 149 & 75,1 & 40,75 min \\
Realizam atividades vigorosas & 13 & 26,15 & 80 min \\
Realizam atividades moderadas & 35 & 71,42 & 125,50 min \\
Realizam atividades leves & 49 & 100,00 & \\
\hline (02) Atividade física como meio de transporte & & & 22,15 min \\
\hline Total de pessoas que se locomovem & 195 & 98,5 & - \\
Caminhada / bicicleta* & 79 & 38,31 & - \\
Veículos automotores & 179 & 90,42 & - \\
Sim* & 61 & 30,81 & 27 min \\
Não & 137 & 69,19 & 30 min \\
Realizam atividades vigorosas & 14 & 22,95 & 100 \\
Realizam atividades moderadas e leves & 61 & &
\end{tabular}

Legenda: * Válidos como atividade física, segundo o IPAQ. Fonte: Freitas RM, et al., 2019.

Quando se avaliou a modalidade dos exercícios físicos (propriamente dita, como lazer ou atividade de recreação), constatou-se que 78 dos 198 participantes da pesquisa demandam efetivamente um tempo à prática desses. Dentre os praticantes, é válido ressaltar que 09 praticam mais de um exercício ao longo da semana. A média de prática efetiva de exercícios físicos foi de $32 \mathrm{~min} / \mathrm{dia}$, em 2,3 dias da semana. 
Em sua última sessão, o IPAQ estima a quantidade de tempo gasto sentado, em atividades passivas (como ver televisão, utilizar o computador ou o celular) durante um dia da semana e nos dias de fim de semana. Dentre os 198 pacientes, 170 (86,20\%) afirmaram permanecer mais de 5 horas/dia sentados, durante a vigília, em um dia de semana. Esse valor aumenta para 190 (96,16\%) quando analisado um dia do fim de semana. A partir da avaliação das sessões, torna-se possível categorizar os pacientes, conforme proposto pelo IPAQ, considerando a frequência e a duração de suas atividades (Tabela 3 ).

Tabela 3 - Categorização, proposta pelo IPAQ, do nível de atividade física praticada, entre idosos assistidos pela Estratégia de Saúde da Família, n=198. Montes Claros - MG, 2017.

\begin{tabular}{lcc}
\hline \multicolumn{1}{c}{ Variável } & N & \% \\
\hline \multicolumn{1}{c}{ Categoria do nível de atividade física } & $\mathbf{1 9 8}$ & $\mathbf{1 0 0}$ \\
\hline Sedentário & 3 & 1,51 \\
Insuficientemente ativo (A) & 129 & 65,15 \\
Insuficientemente ativo (B) & 37 & 18,39 \\
Ativos & 25 & 12,27 \\
Muito ativos & 4 & 2,68 \\
\hline
\end{tabular}

Fonte: Freitas RM, et al., 2019.

\section{DISCUSSÃO}

Ao comparar as variáveis encontradas e apresentados com os resultados encontrados no estudo de Moreschi C, et al. (2015), há uma concordância na predominância de pessoas acima de 60 anos $(60 \%$ em 2013) e do sexo feminino (63,7\% em 2013). Complementando com o trabalho de Assunção SC, et al. (2017), manteve-se predominância do sexo feminino $(73,1 \%)$, cor de pele parda $(53,3 \%)$ e idade de 51 a 60 anos $(28,4 \%)$ e de 61 a 80 anos (50\%), esperado, haja visto que majoritariamente os DM tipo 2, é comumente encontrado em pessoas a partir da meia-idade ( $5^{\underline{a}}$ década de vida, adiante), trazendo proporções compatíveis entre os estudos citados. Ressalta-se que os dados do tipo do DM foi correspondente aos das Diretrizes da Sociedade Brasileira de Diabetes (DSBD, 2017), que enfatiza prevalência do DM tipo 1 em 5 a 10\% dos casos de DM no Brasil, com predomínio do diagnóstico em crianças e adolescentes, e por sua vez, o DM tipo 2, representando 90 a 95\%, com diagnóstico predominantemente a partir dos 40 anos de idade.

Nos últimos seis meses, 76,3\% (151) dos idosos receberam informações adequadas sobre os benefícios da prática de atividades físicas, seja de profissionais da saúde (médicos, enfermeiros, cirurgiões dentistas, agentes comunitários de saúde e educadores físicos), seja por materiais impressos (revista, jornal, outdoor, panfleto, folder, cartaz e cartilha) ou eletrônicos (internet, vídeo educativo, televisão e rádio). O maior percentual de origem das informações entre os participantes foi por profissionais da saúde, que correspondeu a $98 \%$ (148), seguido de materiais eletrônicos com 22,6\% (34) e, por fim, materiais impressos 6,89\% (10), sendo que a maioria dos participantes recebeu formas combinadas de informações. Contudo, apesar da cobertura significativa de participantes, apenas $31,15 \%$ colocaram em prática as recomendações que lhes foram repassadas.

De acordo com os resultados encontrados na seção 2 do IPAQ, "Atividade física como meio de transporte", 179 participantes relataram que utilizava veículos automotivos (carro, ônibus e motocicletas) como meios físicos para se locomover de um local para outro. Houve o relato de 9 participantes que utilizam da bicicleta, por mais de 10 minutos, como meio de locomoção, numa frequência de 4,1 dias por 23 minutos/dia, bem como aqueles que se locomovem pela caminhada, por mais de 10 minutos, correspondendo a 70 participantes, com média de caminhada de $21 \mathrm{~min} /$ dia por 4 dias da semana. É válido ressaltar que 2 dos 198 pacientes são acamados, impossibilitados de se locomoverem.

Conforme resultados da seção 3 do IPAQ, sobre a "Atividade física em casa", abrangendo o cuidar da casa, as tarefas domésticas e o cuidar da família, dentre os 198 participantes, 61 realizam atividades físicas em casa, dos quais 14 realizam atividades vigorosas no jardim ou no quintal, como carpir, lavar e esfregar o 
chão. Foi computado gasto de 2,2 dias na semana e média de $27 \mathrm{~min} /$ dia para as atividades. De acordo com os dados, podemos ver que 61 participantes realizam atividades moderadas e leves, como carregar pesos leves, limpar vidros, varrer e rastelar, com tempo total semanal gasto de 61 minutos.

Na população analisada, há uma discrepância entre o número total de idosos ativos de forma insatisfatória (Sedentários, Insuficientemente ativo (A) e Insuficientemente ativa (B)) e o número de idosos com desempenho satisfatório (Ativo e Muito Ativo), representada percentual e respectivamente em 85,05\% (169) e 14,95\% (29). Ao se considerar todas as modalidades das práticas de atividade física, apenas um sexto dos participantes as realizam em tempo ou frequência suficientes. A inatividade física propicia um pior prognóstico, visto que a atividade física é fator importante para a proteção e controle da progressão do DM (FLOR LS e CAMPOS MR, 2017).

Uma análise mais aprofundada revela que apenas 78 pacientes dos $198(38,31 \%)$ organizam um tempo à prática de exercícios físicos, com tempo médio gasto de 73,5 min/semana. Além da baixa adesão dos idosos com DM avaliados aos exercícios, aqueles que os praticam o fazem por tempo inferior ao preconizado pelas Diretrizes da Sociedade Brasileira de Diabetes, que indica exercício aeróbico moderado com duração de 150 minutos por semana, exercício aeróbico intenso de 75 minutos por semana ou a combinação das duas intensidades, somados a exercícios de fortalecimento muscular, no mínimo duas vezes por semana (DSBD, 2017).

Apenas 78 dos 198 idosos (38,31\%) entrevistados relatam a prática efetiva de exercícios físicos, o que corrobora com a visualização de valores expressivos (superior a 50\%) de pessoas com DM ainda sedentárias, mesmo diante da eficácia da prática de exercícios como coadjuvante no controle do DM. Comparando a outro estudo que estimou o nível de exercícios físicos praticados em pessoas com DM tipos 1 e 2, percebeu-se que 225 pessoas somente $85(37,7 \%, \mathrm{n}=225)$ praticavam exercícios físicos regularmente e $87(38,8 \%)$ realizavam autocuidados como alimentação, alongamento e/ou monitoramento da glicemia capilar (DUARTE CK, et al., 2012).

Os valores encontrados estabelecem perfil de semelhança, visto que já tenha sido observado a melhora na glicemia de jejum, na hemoglobina glicada ( $\mathrm{HbA1c})$, nos níveis lipídios plasmáticos, na frequência cardíaca de repouso e no índice de massa corporal, após uma sessão aguda de exercício aeróbico, resistido e de flexibilidade (na mesma sessão) em pessoas com DM tipo 2. E que o efeito hipoglicemiante como resposta do exercício físico em pessoas com DM tratados ou não com insulina. Explicita-se assim a importância do exercício físico para as pessoas com DM (SILVA CA e LIMA WC, 2002).

As pessoas com DM tipo 1 necessitam de instrução individualizada, de acordo com suas necessidades, sendo importante o acesso a carboidrato de rápida absorção, para que seja consumido em caso de hipoglicemia e aqueles sem outras morbidades que impeçam a prática de atividade física e que possuam um bom controle glicêmico, estão aptos a realizar todos os níveis de exercício físico, como de lazer, esporte recreacional e profissional (GIORELLI GV, et al., 2015).

Dentre os 198 idosos, 151 receberam informações atualizadas (76,3\%) sobre os benefícios da prática de exercícios, sendo que entre 148 (98\%) essas informações foram repassadas por profissionais da saúde. Outro estudo evidenciou que pessoas com DM sem atendimento em Unidades Básicas de Saúde apresentavam pouco conhecimento sobre a doença, reduzindo o envolvimento destes em seu tratamento, concluindo a necessidade de mudanças nas atividades de educação em saúde voltadas para motivação das pessoas com DM e da sociedade em geral (ASSUNÇÃO SC, et al., 2017). Pois, mesmo em um estudo que se observou que várias pessoas com DM obtiveram bom escore de conhecimento não modificaram a atitude para enfrentarem a doença de forma mais adequada (RODRIGUES FFL, et al., 2012), assemelhando-se aos dados registrados entre os idosos, pois somente $47(31,15 \%, n=151)$ colocaram em prática as recomendações que Ihes foram repassadas.

Sendo assim, uma vez que já foi evidenciada a queda de glicemia sérica no período da prática de exercícios em cerca de 50mg/dl após 45 minutos no trabalho de Marçal DFS, et al. (2018), torna-se imprescindível a estimulação das pessoas com DM para a efetiva prática de atividades físicas, respeitando suas individualidades, a fim de incrementar em sua terapêutica uma medida tão eficaz no controle glicêmico, bem como de outras morbidades que podem agravar o DM. 


\section{CONCLUSÃO}

Buscou-se descrever o nível de conhecimentos dos idosos portadores de DM na Atenção Primária acerca da importância e da efetiva prática de atividades físicas, sabidamente importante como medida profilática e terapêutica ao adequado controle glicêmico, assim como de outras morbidades dentre elas as principais síndromes geriátricas. Por meio do IPAQ, avaliou-se a frequência e a modalidade das atividades físicas em diferentes contextos: no ambiente de trabalho, no transporte, em casa, como recreação e, ainda, o tempo gasto em atividades passivas. O estudo evidenciou que a maioria dos idosos é insuficientemente ativa, ou seja, pratica exercícios durante a semana, entretanto com frequência e/ou duração insuficiente para serem classificados como pessoas fisicamente ativas, ainda que a maioria relatou ter recebido informações sobre os benefícios das atividades físicas no controle do DM assim como de outras morbidades. Evidencia-se portanto a necessidade de melhorias na cobertura por parte da Atenção Primária no que tange ao repasse e à aplicabilidade da prática saudável de atividade física como estratégia adjuvante do controle glicêmico.

\section{REFERÊNCIAS}

1. ASSUNÇÃO SC, et al. Conhecimento e atitude de pacientes com diabetes mellitus da Atenção Primária a Saúde. Escola Anne Nery Revista de Enfermagem. Rio de Janeiro - Rio de Janeiro. 2017; 21(4): 1-7.

2. COLBERG SR, et al. Atividade física/exercício e diabetes: a posição da Associação Americana de Diabetes. Diabetes Care. 2016; 39: 2065-2079.

3. DE LADE CG, et al. Efeitos de diferentes programas de exercícios e alterações mínimas detectáveis na hemoglobina A1c em pacientes com diabetes tipo 2. Diabetologia e Síndrome Metabólica. 2016; 8(13): 1-113.

4. DIRETRIZES DA SOCIEDADE BRASILEIRA DE DIABETES 2017-2018 / Organização José Egídio Paulo de Oliveira, Renan Magalhães Montenegro Junior, Sérgio Vencio. - São Paulo : Editora Clannad, 2017.

5. DUARTE CK et al. Nível de atividade física e exercício físico em pacientes com diabetes mellitus. Rev. Assoc. Med. Bras. [online]. 2012; 58(2): 1215-1221.

6. FILHO CAAD et al. Exercício físico e diabetes. Revista Brasileira de Prescrição e Fisiologia do Exercício. São Paulo - SP, 2016; 10( 57):1-4.

7. FLOR LS, CAMPOS MR. Prevalência de diabetes mellitus e fatores associados na população adulta brasileira: evidências de um inquérito de base populacional. Revista Brasileira de Epidemiologia. Rio de Janeiro - RJ, 2017; 20(1) 16-29.

8. GIORELLI GV, et al. Educação física e diabetes: prevenção e tratamento. Revista HUPE, Rio de Janeiro, 2015; 14(4): 54-58

9. ISER BPM, et al. Prevalência de diabetes autorreferidos no Brasil: resultados da Pesquisa Nacional de Saúde de 2013, Revista Epidemiologia e Serviços de Saúde, Brasília - DF, 2015; 24(2): 305-314.

10. KER RG. Atividade física no combate à Diabetes Mellitus tipo 2. Monografia - Faculdade de Educação Física, Faculdade de Educação e Meio Ambiente, Arquiemes - RO, 2018: 1-29.

11. MALTA DC, et al. Fatores associados ao diabetes autorreferido segundo a Pesquisa Nacional de Saúde, 2013. Revista de Saúde Pública. Belo Horizonte - MG, 2017; 51:1-12.

12. MARÇAL DFS, et al. Efeitos do exercício físico sobre o diabetes mellitus tipo 1: uma revisão sistemática de ensaios clínicos e randomizados. Jornal de Educação Física. Maringá - PR, 2018; 29:1-14.

13. MATSUDO S, et al. Questionário internacional de atividade física (IPAQ): estudo de validade e reprodutibilidade no Brasil. Rev.Bras.Ativ.Fís.Saúde. 2001; 6(2): 5-18.

14. MAZO GZ. Recomendações de atividade físicas para idosos. Rev. Biomotriz, Cruz Alta - RS, Dez./2018; $12(1): 1-4$.

15. MORESCHI C, et al. Prevalência e perfil das pessoas cadastradas no sistema de informação da atenção básica (SIAB). Revista Brasileira em Promoção da Saúde. Fortaleza - Ceará. 2015; 28(2): 184-190.

16. RAMOS RSPS, et al. Fatores associados ao diabetes em idosos assistidos em serviço ambulatorial especializado geronto-geriátrico. Rev. Bras. Geriatr. Gerontol., Rio de Janeiro, 2017; 20(3): 364-374.

17. RODRIGUES FFL, et al. Relação entre conhecimento, atitude, escolaridade e tempo de doença em indivíduos com diabetes mellitus. Acta Paul Enferm. 2012; 25(2): 284-90.

18. SANTOS LM, et al. Avaliação do hábito alimentar e estado nutricional de idosos com diabetes mellitus tipo 2 atendidos na atenção básica de saúde do município de Porteiras-CE. Revista E-Ciência, 2017; 5(1): 69-77.

19. SILVA CA, LIMA WC. Efeito benéfico do exercício físico no controle metabólico do diabetes mellitus tipo 2 a curto prazo. Arq.Bras.Endocrinol.Metabol. 2002; 46(5): 550-6.

20. SILVA GSF, et al . Avaliação do nível de atividade física de estudantes de graduação das áreas saúde/biológica. Rev. Bras. Med. Esporte, Niterói, 2007; 13(1): 39-42.

21. VITOI NC, et al. Prevalência e fatores associados ao diabetes em idosos no município de Viçosa, Minas Gerais. Rev. Bras. Epidemiol. 2015; 18(4): 953-965. 